\title{
(2) OPEN ACCESS \\ Medication-related harm in older adults following hospital discharge: development and validation of a prediction tool
}

\author{
Nikesh Parekh (D) ,' Khalid Ali, ${ }^{1}$ John Graham Davies, ${ }^{2}$ \\ Jennifer M Stevenson, ${ }^{2}$ Winston Banya, ${ }^{3}$ Stephen Nyangoma, ${ }^{3}$ \\ Rebekah Schiff, ${ }^{4}$ Tischa van der Cammen, ${ }^{5}$ Jatinder Harchowal, ${ }^{6}$ \\ Chakravarthi Rajkumar ${ }^{1}$
}

- Additional material is published online only. To view, please visit the journal online (http://dx.doi.org/10.1136/ bmjqs-2019-009587).

${ }^{1}$ Academic Department of Geriatric Medicine, Brighton and Sussex Medical School, Brighton, UK

${ }^{2}$ Institute of Pharmaceutical Science, King's College London, London, UK

${ }^{3}$ Royal Brompton and Harefield NHS Foundation Trust, London, UK

${ }^{4}$ Guy's and St Thomas' NHS Foundation Trust, London, UK ${ }^{5}$ Faculty of Industrial Design Engineering, Delft University of Technology, Delft, The Netherlands

${ }^{6}$ Royal Marsden Hospital NHS Trust, London, UK

\section{Correspondence to} Dr Nikesh Parekh, Academic Department of Geriatric Medicine, Brighton and Sussex Medical School, Brighton BN1 9PX, UK; nikeshparekh@nhs.net

Received 21 March 2019 Revised 20 August 2019 Accepted 29 August 2019 Published Online First

16 September 2019

\section{Check for updates}

\section{(c) Author(s) (or their} employer(s)) 2020. Re-use permitted under CC BY-NC. No commercial re-use. See rights and permissions. Published by BMJ.

To cite: Parekh N, Ali K, Davies JG, et al. BMJ Qual Saf 2020;29:142-153.

\begin{abstract}
Objectives To develop and validate a tool to predict the risk of an older adult experiencing medication-related harm (MRH) requiring healthcare use following hospital discharge.

Design, setting, participants Multicentre, prospective cohort study recruiting older adults ( $\geq 65$ years) discharged from five UK teaching hospitals between 2013 and 2015.

Primary outcome measure Participants were followed up for 8 weeks in the community by senior pharmacists to identify MRH (adverse drug reactions, harm from non-adherence, harm from medication error). Three data sources provided MRH and healthcare use information: hospital readmissions, primary care use, participant telephone interview. Candidate variables for prognostic modelling were selected using two systematic reviews, the views of patients with MRH and an expert panel of clinicians. Multivariable logistic regression with backward elimination, based on the Akaike Information Criterion, was used to develop the PRIME tool. The tool was internally validated.

Results 1116 out of 1280 recruited participants completed follow-up (87\%). Uncertain MRH cases ('possible' and 'probable') were excluded, leaving a tool derivation cohort of $818.119(15 \%)$ participants experienced 'definite' MRH requiring healthcare use and 699 participants did not. Modelling resulted in a prediction tool with eight variables measured at hospital discharge: age, gender, antiplatelet drug, sodium level, antidiabetic drug, past adverse drug reaction, number of medicines, living alone. The tool's discrimination C-statistic was 0.69 (0.66 after validation) and showed good calibration. Decision curve analysis demonstrated the potential value of the tool to guide clinical decision making compared with alternative approaches.

Conclusions The PRIME tool could be used to identify older patients at high risk of MRH requiring healthcare use following hospital discharge. Prior to clinical use we recommend the tool's evaluation in other settings.
\end{abstract}

\section{BACKGROUND}

Reducing the burden of serious and avoidable medication-related harm (MRH) by $50 \%$ by 2022 is WHO's third global patient safety challenge. ${ }^{1}$ Transitions of care, particularly following hospital discharge, ${ }^{2}$ present a heightened risk and addressing this is a priority. ${ }^{3}$ Medicationrelated harm includes harm from adverse drug reactions (ADR), non-adherence and medication errors. ${ }^{2}$

Older adults are especially vulnerable to experience MRH due to multimorbidity and polypharmacy, ${ }^{4}$ age-related changes in pharmacokinetics and pharmacodynamics ${ }^{5}$ and adherence problems. ${ }^{6}$ Patients and carers identify the care transition around hospital discharge as a unique, high-risk period for the occurrence of $\mathrm{MRH}^{7-9}$ Contributory factors include deconditioning during hospitalisation, ongoing recovery from acute illness,${ }^{10}$ inaccuracies in medicines reconciliation, ${ }^{11}$ insufficient patient education of medicines use ${ }^{12}$ and poor communication between secondary and primary care. $^{1314}$ A systematic review of MRH in older adults found that between 17\% and $51 \%$ of patients experience $\mathrm{MRH}$ within 30 days of hospital discharge. ${ }^{2}$ In the UK, $28 \%$ of older adults ( $\geq 65$ years) use health services due to MRH within the 8 weeks following hospital discharge, at a cost of $£ 400$ million (US $\$ 530$ million) to the National Health Service (NHS) annually. ${ }^{15}$

Current strategies to address MRH during transitions of care include medicines review incorporating a patientcentred approach to deprescribing ${ }^{16} 17$ 
and the improvement of information transfer through medicines reconciliation. ${ }^{18}$ Risk stratification using prediction tools is recognised as one solution to enhance the cost-effectiveness of interventions by identifying patients likely to derive greatest benefit. ${ }^{19}{ }^{20}$ Individual risk prediction can inform clinical decision making and support patient participation in their healthcare. Risk prediction tools such as the Framingham ${ }^{21}$ or QRISK $^{22}$ tools for estimating cardiovascular risk have transformed the provision of healthcare.

Currently, no tools are available to target intervention to patients at high risk of $\mathrm{MRH}$ in the community following hospital discharge. ${ }^{23}$ Relying on clinical judgement alone is poorly predictive. ${ }^{24} \mathrm{~A}$ key recommendation in a position statement from the International Group for Reducing Inappropriate Medication Use and Polypharmacy is to 'develop tools that can aid the detection and management of drug adverse effects'. ${ }^{25}$

The aim of our study was to develop and validate a risk prediction tool to identify older patients at high risk of MRH requiring healthcare use within 8 weeks following hospital discharge.

\section{METHODS}

\section{Design, setting and participants}

The risk prediction tool reported in this paper was developed using data from a multicentre, prospective cohort study; Prospective study to develop a model to stratify the RIsk of Medication-related harm in hospitalised Elderly patients (PRIME). ${ }^{15} 26$ Detailed methods are in the published protocol. ${ }^{26}$ This study is reported in accordance with the transparent reporting of a multivariable prediction model for individual prognosis or diagnosis (TRIPOD) statement. ${ }^{27}$

Research nurses recruited inpatients (age $\geq 65$ years) close to discharge from medical wards, between 2013 and 2015, in five NHS teaching hospitals in England. Patients were excluded if they were terminally ill, if they lacked capacity with no nominated consultee or if they were transferred to other acute healthcare units.

\section{Baseline data collection}

Research nurses collected baseline information from consenting patients using medical records and patient interview, including demographic, clinical and social data.

\section{Outcome definition}

The risk prediction model was developed to predict $\mathrm{MRH}$ requiring healthcare within 8 weeks of hospital discharge. MRH was defined as an ADR or harm arising from non-adherence. Non-adherence was defined as failure to use medicines as the prescriber intended. Harm arising from medication error was included where reported. Intentional overdose was excluded. Asymptomatic derangements in blood markers were not included as MRH. This is a modified version of the definition by Strand $e t a l .{ }^{28}$

Healthcare use included primary, secondary or tertiary consultations related to MRH. Senior, research pharmacists followed participants for 8 weeks to determine MRH occurrence. An 8-week observation period was chosen as previous research outside of the UK had found this is a reasonable timeframe to capture most postdischarge MRH. ${ }^{29-31}$ Classification of MRH events was completed using the validated Naranjo algorithm as 'possible', 'probable' or 'definite'. ${ }^{32-35}$ Where an event was not suspected, this was classified as 'doubtful'. To eliminate uncertainty and strengthen the external validity of the model, any events that were possible or probable were excluded. Only events that required healthcare use were included as an objective threshold for MRH severity.

\section{MRH and healthcare use assessment}

Pharmacists identified MRH and healthcare use (accident and emergency department attendance, hospital admission, outpatient clinics, general practitioner (GP) in-person or telephone consultations and out-of-hours care) from three sources: (1) participant/carer telephone interview at 8 weeks using a structured questionnaire, (2) GP records and (3) prospective review of hospital readmissions with the admitting medical consultant.

The Naranjo Algorithm ${ }^{35}$ was used to assess ADR causality in conjunction with the British National Formulary and Summary of Product Characteristics. Clinical judgement supported by a validated questionnaire $^{36}$ and prescription order data were used to determine if non-adherence had contributed to MRH.

Two senior study pharmacists provided case-based training to all other pharmacists. Additionally, crosssite case discussions were held to ensure standardisation of MRH assessments.

An end point committee independent from data collection, consisting of a professor of geriatrics, professor of clinical pharmacy and two consultant geriatricians reviewed, scrutinised and verified MRH cases.

\section{Selection of candidate predictors}

Candidate variables for the PRIME tool were prospectively selected by (1) two systematic reviews exploring previous MRH risk prediction tools, and, the epidemiology of MRH postdischarge, ${ }^{23}$ (2) three round-table expert meetings to identify candidate predictors, prior to any data analysis, based on clinical relevance and practicality of routine measurement (including two professors of geriatrics, two consultant geriatricians, one professor of pharmacy, one hospital chief pharmacist, one pharmacy research fellow and one geriatrics research fellow), (3) a qualitative study to identify MRH risk factors from the patient and carer perspective. ${ }^{37}$ At this initial stage no upper limit was placed on 
the number of candidate variables. ${ }^{26} 38$ All candidate variables were selected based on clinical relevance and not using univariable analysis to avoid the possibility of variable inclusion by statistical chance. ${ }^{3940}$ Once the number of MRH events in the study was known, the number of variables was reduced for model development recognising 10 events per variable by Peduzzi et $a l$ as a rough indicator. ${ }^{38}$ However, the use of this 'rule of thumb' is not universally advocated. ${ }^{4142}$

\section{Sample size}

The study was adequately powered based on a calculation using the nomogram designed by Carley et $a l^{43}$ and based on the work of Buderer et al. ${ }^{44} \mathrm{~A}$ sample size calculation was performed to achieve a sensitivity of $80 \%$ with a $95 \%$ CI width of $5 \%$ and based on an MRH prevalence rate of $30 \%$ (see online supplementary file for additional detail).

\section{Missing data}

Under a missing at random assumption, we imputed data on all variables with missing data; estimated glomerular filtration rate, hand grip strength, past $\mathrm{ADR}$ and living alone postdischarge. These values were replaced using Multiple Imputation by Chained Equations. ${ }^{45}$ For each variable 10 imputed datasets were created and Rubin's rule was used to obtain an overall estimate. ${ }^{45}$ See online supplementary file for additional detail.

\section{Statistical analysis}

All statistical analyses were conducted in Stata V.14 (StataCorp, College Station, Texas, USA).

\section{Model development}

The primary outcome was whether a person experienced MRH requiring healthcare use; a binary variable. Backward elimination, based on the Akaike Information Criterion (AIC), excluded variables that were not contributing sufficient predictive information in the multivariable logistic regression model ${ }^{46}$ (the AIC is a predictive metric that equates to the elimination of variables with $\mathrm{p} \geq 0.157$ ). The AIC was chosen as the selection procedure as it balances the risk of overfitting a model to the derivation data and introducing misspecification error from trimming potentially meaningful variables, while penalising the inclusion of redundant variables contributing little predictive information to generate a parsimonious model. ${ }^{47}$ Two variables (age and sodium level) were centred on their mean values to make the interpretation of the intercept easier and meaningful. In the model equation, the intercept can be interpreted as the predicted risk of $\mathrm{MRH}$ if all the continuous explanatory variables are set to 0 (and the categorical variables held at their baseline values), a value which is infeasible for continuous variables such as age and sodium level. Therefore by centring these two variables, the intercept in the model equation would indicate the predicted risk of MRH with plausible values for age and sodium level. Two-way interactions between pairs of variables were tested and no significant interactions were found between them in their relationship with MRH.

A final model of eight predictors was obtained after backward elimination and a risk equation for predicting the $\log$ odds of MRH was formed by summing the products of the estimated $\beta$-coefficients (obtained from fitting the multivariable logistic regression model) and their corresponding observed predictors, plus the intercept.

All the predictors were assessed for collinearity by calculating the variance inflation factor (VIF) to identify whether variances of the estimated coefficients were inflated. ${ }^{48}$

\section{Evaluating model performance}

The performance of the model was evaluated by its discrimination (area under the receiver operating characteristic curve) and calibration (calibration slope and Hosmer-Lemeshow goodness-of-fit statistic). ${ }^{49}$

Bootstrap correction of model optimism and validation

Bootstrap is a model validation approach that is recommended when an external cohort of patients is unavailable to estimate the performance of the prediction model. ${ }^{50-53}$ Bootstrap is a resampling method that is used to randomly generate data (data for subsets of patients) from the original sample with replacement (patients can be selected multiple times). The bootstrap sample is the same size as the original sample $(n=818)$. One hundred samples of the derivation data were bootstrapped, and, in each sample a prediction model was developed and used to compute an estimate of model optimism (see online supplementary file for additional detail).

\section{Assessment of potential clinical usefulness}

A net benefit approach using decision curve analysis was conducted to explore the potential clinical utility of the PRIME tool. This type of analysis measures the benefits and harms (in terms of true positives and false positives) of using the tool to guide intervention, ${ }^{54}$ that is, in the current context, intervention to avoid MRH. A decision analytic curve can compare the net benefit of using this new approach with existing approaches to guide intervention, for example, polypharmacy, ${ }^{16} 17$ or an approach of treating all people of a particular risk threshold, or alternatively treating none. The 'threshold' risk at which a clinician would decide to intervene is subjective and should incorporate patient preferences. Furthermore, the threshold will depend on the time and cost resource, invasiveness, effectiveness and harms of the intervention. ${ }^{545}$ However, a reasonable range of threshold probabilities in the context of intervening on the risk of serious MRH was considered to be $5 \%-25 \%$. This range reflects expert 
Table 1 Baseline sample characteristics

\begin{tabular}{|c|c|c|c|}
\hline $\begin{array}{l}\text { Key characteristics, } \\
\text { n (\%) unless otherwise stated }\end{array}$ & No MRH $(n=699)$ & $\begin{array}{l}\text { MRH (possible, probable, } \\
\text { definite) }(n=413)\end{array}$ & $\begin{array}{l}\text { MRH definite requiring } \\
\text { healthcare }(n=119)\end{array}$ \\
\hline Age, mean (SD), years & $80.9(7.9)$ & $81.7(7.4)$ & $82.6(6.5)$ \\
\hline Female* & $383(54.8)$ & $268(64.9)$ & $81(68.1)$ \\
\hline Length of hospital stay, median (IQR), days & $6(3-13)$ & $8(4-14)$ & $7(3-14)$ \\
\hline \multicolumn{4}{|l|}{ Charlson Comorbidity Index } \\
\hline $0-1$ & $342(48.9)$ & $198(47.9)$ & $56(47.1)$ \\
\hline$\geq 2$ & $357(51.1)$ & $215(52.1)$ & $63(52.9)$ \\
\hline Renal impairment† (eGFR <60) & $260(40.0)$ & $170(44.4)$ & $59(52.7)$ \\
\hline Barthel score*, median (IQR) & $18(13-20)$ & $17(13-19)$ & $17(13-19)$ \\
\hline \multicolumn{4}{|l|}{ Hand grip $\neq$, median (IQR), kg } \\
\hline Female & $13.5(10.0-18.3)$ & $14.0(10.0-18.0)$ & $14.0(10.0-18.0)$ \\
\hline Male & $24.0(19.0-31.0)$ & $24.0(17.8-31.0)$ & $25.0(18.5-30.0)$ \\
\hline Number of medicines*, mean (SD) & $8.9(4.1)$ & $10.0(4.0)$ & $10.4(4.1)$ \\
\hline Number of new medicines, mean $(S D)^{*}$ & $3.1(2.3)$ & $3.5(2.3)$ & $3.4(2.2)$ \\
\hline Past adverse drug reaction ${ }^{\star} \S$ & $202(29.1)$ & $145(35.4)$ & $50(42.4)$ \\
\hline $\begin{array}{l}\text { Medication compliance aid } \\
\text { (eg, dosette box) }\end{array}$ & $224(32.0)$ & $146(35.4)$ & $50(42.0)$ \\
\hline Living alone after discharge* & $322(46.2)$ & $228(55.5)$ & $69(58.0)$ \\
\hline
\end{tabular}

*Significant difference between 'MRH' and 'no MRH' groups: female, $p=0.001$; Barthel score, $p=0.012$; number of medicines, $p<0.001$; past adverse drug reaction, $p=0.037$; living alone after discharge, $p=0.003$.

tInformation not available for 79 participants.

łInformation not available for 117 participants.

§Information not available for 9 participants.

IInformation not available for 4 participants.

eGFR, estimated glomerular filtration rate $\mathrm{mL} / \mathrm{min} / 1.73 \mathrm{~m}^{2} ; \mathrm{MRH}$, medication-related harm.

consensus opinion of the multidisciplinary PRIME study coordinating team. The assumption here that it would be surprising if a patient had $<5 \%$ risk of serious MRH and a decision was made to intervene, that is, an acceptance of intervening on 19 patients unnecessarily to avoid one patient experiencing MRH. Likewise, if a patient has $>25 \%$ (one in four) chance of experiencing serious MRH it would be a surprising decision to take no action to mitigate this risk.

\section{RESULTS}

\section{Participant characteristics}

A total of 1280 older adults were recruited at hospital discharge for 8-week follow-up. Seventeen participants died without follow-up (1.3\%), 147 participants $(11.5 \%)$ were lost to follow-up because they were uncontactable and GP records were unavailable (see online supplementary file for further detail and participant flow chart). One hundred nineteen participants experienced 'definite' MRH requiring healthcare use within 8 weeks following hospital discharge (an incidence of 107 participants per 1000 discharged). The model derivation cohort included 818 participants with either 'definite' MRH requiring healthcare use or no MRH requiring healthcare use (table 1). The 'possible' and 'probable' cases of MRH, as well as 'definite' cases that did not require healthcare use were excluded.

\section{Model development and performance}

Twenty-five candidate variables were initially identified based on clinical judgement, existing literature and qualitative work with patients and carers. Three of the 25 variables (albumin level, C reactive protein, white cell count) were excluded due to substantial missing data (>20\%). Two variables (change in accommodation after discharge and hepatic impairment) were excluded due to insufficient prevalence in the cohort $(<10 \%)$. One variable (cardiovascular drug on discharge) was excluded due to saturated prevalence in the cohort $(>80 \%)$. The remaining 19 variables were taken forward for potential inclusion in the final multivariable analysis, and 12 predictor combinations were trialled to obtain a parsimonious model with optimal performance and stability (broadly adhering to the 10 events-per-variable ratio rule of thumb ${ }^{38}$ to reduce model performance bias ${ }^{56}$ ). Models were examined for evidence of multicollinearity, and where demonstrated the variable contributing the least predictive value was excluded. The iterative procedure resulted in the exclusion of a further seven variables (regular falls, Barthel score, addition of a new drug, opiate drug, anticoagulant drug, abbreviated mental test score, depression on screening). The 12 predictors included in a final multivariable model to generate the PRIME tool are shown in table 2 (see online supplementary file for summary of excluded variables). 
Table 2 Selected candidate predictors to derive the risk prediction model

\begin{tabular}{|c|c|c|c|c|c|c|}
\hline Variable & Data source and measurement & Prevalence* & $\begin{array}{l}\text { Unadjusted } \\
\text { OR }(95 \% \mathrm{Cl}) \text { on } \\
\text { univariable analysis }\end{array}$ & $\begin{array}{l}\text { Adjusted OR }(95 \% \mathrm{CI}) \text { multivariable } \\
\text { regression based on backwards } \\
\text { elimination based on Akaike } \\
\text { Information Criterion ( } \mathrm{p}>0.157)\end{array}$ & $\begin{array}{l}\boldsymbol{\beta} \text {-Coefficients } \\
\text { of variables } \\
\text { included in } \\
\text { model }\end{array}$ & $\begin{array}{l}P \text { value in } \\
\text { multivariable } \\
\text { analysis }\end{array}$ \\
\hline Age (years) & Self-report and medical records & 81.2 & $1.03(1.00$ to 1.06$)$ & $1.03(1.00$ to 1.05$)$ & 0.025 & 0.078 \\
\hline Gender (reference female) & Self-report and medical records & $\begin{array}{l}43.3 \text { (male) } \\
56.7 \text { (female) }\end{array}$ & 0.57 (0.38 to 0.86$)$ & 0.67 (0.43 to 1.04$)$ & -0.398 & 0.075 \\
\hline Past ADR† & Self-report and medical records & $31.1 \%$ & 1.79 (1.20 to 2.67$)$ & 1.61 (1.06 to 2.45$)$ & 0.477 & 0.026 \\
\hline Antiplatelet drug & $\begin{array}{l}\text { Discharge summary and medical } \\
\text { records. Drugs coded B01AC on WHO- } \\
\text { ATC system }\end{array}$ & $43.3 \%$ & 1.78 (1.20 to 2.63$)$ & 1.67 (1.11 to 2.53$)$ & 0.515 & 0.014 \\
\hline Antidiabetic drug & $\begin{array}{l}\text { Discharge summary and medical } \\
\text { records. Drugs coded A10A or A10B on } \\
\text { WHO-ATC system }\end{array}$ & $19.7 \%$ & 1.89 (1.22 to 2.94$)$ & 1.81 (1.12 to 2.91$)$ & 0.591 & 0.016 \\
\hline Living alonet & Self-report and medical records & $47.9 \%$ & 1.61 (1.08 to 2.38$)$ & 1.49 (0.98 to 2.27$)$ & 0.397 & 0.064 \\
\hline Sodium level (mmol/L) & $\begin{array}{l}\text { Last recorded inpatient biochemistry } \\
\text { prior to discharge }\end{array}$ & 137 & 0.97 (0.93 to 1.01$)$ & $0.96(0.92$ to 1.00$)$ & -0.042 & 0.069 \\
\hline Number of medicines & $\begin{array}{l}\text { Discharge summary and medical } \\
\text { records. Total number of medicines at } \\
\text { discharge }\end{array}$ & 9 & 1.08 (1.04 to 1.13$)$ & $1.06(1.00$ to 1.11$)$ & 0.056 & 0.033 \\
\hline Hand grip strength $(\mathrm{kg}) \dagger$ & $\begin{array}{l}\text { Southampton Protocol for Adult Grip } \\
\text { strength Measurement using the } \\
\text { JAMAR Hydraulic Hand Dynamometer }{ }^{93}\end{array}$ & $\begin{array}{l}25.6 \text { (male) } \\
14.3 \text { (female) }\end{array}$ & $0.98(0.96$ to 1.00$)$ & Eliminated from model $(p>0.157)$ & N/A & 0.911 \\
\hline Medication compliance aid & $\begin{array}{l}\text { Multicompartment compliance aid on } \\
\text { discharge }\end{array}$ & $33.5 \%$ & 1.54 (1.03 to 2.29$)$ & Eliminated from model $(p>0.157)$ & $N / A$ & 0.302 \\
\hline $\begin{array}{l}\text { Renal impairment (eGFR } \\
<60 \mathrm{~mL} / \mathrm{min} / 1.73 \mathrm{~m}^{2)} \dagger\end{array}$ & $\begin{array}{l}\text { Last recorded inpatient biochemistry } \\
\text { prior to discharge }\end{array}$ & $41.9 \%$ & 1.67 (1.13 to 2.47$)$ & Eliminated from model $(p>0.157)$ & N/A & 0.216 \\
\hline Charlson Comorbidity Index & $\begin{array}{l}\text { Discharge summary and medical } \\
\text { records } \\
\text { Total number of diseases defined by } \\
\text { Charlson Comorbidity Index }\end{array}$ & $\begin{array}{l}48.7 \%(<2) \\
51.3 \%(\geq 2)\end{array}$ & $1.12(0.95$ to 1.31$)$ & Eliminated from model $(p>0.157)$ & $\mathrm{N} / \mathrm{A}$ & 0.403 \\
\hline
\end{tabular}

Backward elimination using the AIC (equating to $\mathrm{p}=0.157)^{46}$ resulted in a final risk prediction model of eight variables: age, gender (female $=0)$, antiplatelet drug (antiplatelet on discharge $=1$ ), sodium level, antidiabetic drug (antidiabetic on discharge $=1$ ), history of $\mathrm{ADR}$ (past $\mathrm{ADR}=1$ ), number of discharge medicines and living alone (living alone postdischarge $=1$ ). The final model equation along with case examples to illustrate use of the prediction tool is shown in figure 1.

The linear relationship between continuous predictors (modelled on their continuous scales) and the logodds of the outcome variable was assessed by the use of multivariable fractional polynomials. A Wald test showed that there was a linear relationship between the continuous variables and the log-odds of the outcome. No multicollinearity was demonstrated in this model (mean VIF=1.07). The apparent discrimination of the model was AUC 0.69 (95\% CI 0.64 to 0.74) (figure 2). For comparison, the number of medicines alone discriminated between higher and lower risk patients with AUC 0.61 (95\% CI 0.55 to 0.66 ). A sensitivity analysis found a poorer model discrimination if 'possible' and 'probable' MRH were categorised with 'definite' MRH (C-statistic $0.64,95 \%$ CI 0.60 to $0.67)$, or if 'probable' events were grouped with 'definite' MRH (C-statistic 0.65 , 95\% CI 0.61 to 0.69 ).

The optimism of the model's discriminatory performance was 0.028 , calculated as the average difference between model discrimination in the bootstrap sample and the discrimination of the bootstrap model in the original sample over 100 bootstrap iterations. The optimism adjusted model discrimination is AUC 0.66 (95\% CI 0.61 to 0.71 ).

The calibration, that is, the level of agreement between predicted and observed risk probabilities was good (see online supplementary file for calibration plot). The Hosmer-Lemeshow goodness-of-fit statistic was 5.47 , with $8 \mathrm{df}, \mathrm{p}=0.71$ indicating no evidence of statistically significant difference between the observed and expected values. A uniform shrinkage factor of 0.85 , derived from the average of the calibration slopes of the bootstrap iterations, was applied to predictor coefficients to adjust the risk prediction model for optimism. Figure 3 shows the results of the decision curve analysis, which demonstrates the superior net benefit that use of the PRIME tool offers compared with alternative approaches of 'treat all', 'treat none' or using a binary polypharmacy model to aid clinical decision making over a range of threshold probabilities of approximately 5\%-40\%.

\section{DISCUSSION}

The PRIME tool is the first validated tool to predict the absolute risk of an older person experiencing medication harm in the postdischarge period; the risk of $\mathrm{MRH}$ in this transition period is approximately 
Model equation for risk score $=-2.384+0.85 \times(0.025$ (age-81) -0.398 (gender) +0.515 (antiplatelet drug) 0.042 (sodium-137) +0.591 (antidiabetic drug) +0.477 (past ADR) +0.056 (number of medicines) +0.397 (living alone)

Individual estimated risk of $M R H(\%)=\left(1 / 1+e^{-r i s k ~ s c o r e ~}\right) * 100$

Case Example 1.

$\mathrm{Mr} \mathrm{B}$ is aged 85 years and was hospitalised for a community-acquired pneumonia. He lives with his daughter in a bungalow. He has a medical history of a stent following a myocardial infarction, hypertension, osteoarthritis and benign prostatic hypertrophy. His discharge medications include clopidogrel, atorvastatin, atenolol, ramipril, tamsulosin, paracetamol, codeine phosphate, macrogol and 4 days of co-amoxiclav to complete an antibiotic course. His last sodium level was $130 \mathrm{mmol} / \mathrm{L}$.

Risk score $=-2.384+0.85 \times(0.025(85-81)+-0.398(1)+0.515(1)+-0.042(130-137)+0.591(0)+0.477(0)+$ $0.056(9)+0.397(0)$

Individual estimated risk of $\operatorname{MRH}(\%)=\left(1 / 1+\mathrm{e}^{-1.520}\right) * 100$

Mr B's absolute risk of experiencing MRH requiring healthcare use over the next 8 weeks is estimated at $18 \%$

Case Example 2.

Mrs V is aged 78 years and was hospitalised following a fall and fractured neck of femur. She lives alone in sheltered accommodation. She has a past medical history of poorly controlled type 2 diabetes, hypertension, moderate stage chronic kidney disease and depression. Her discharge medications include gliclazide, sitagliptin, doxazosin, losartan, mirtazapine, zopiclone, paracetamol, oxycodone, senna, adcal D3, alendronate, and omeprazole. Her last sodium level was $132 \mathrm{mmol} / \mathrm{L}$. Mrs V recalls seeing her GP with swollen ankles last year, which improved after her amlodipine was stopped.

Risk score $=-2.384+0.85 \times(0.025(79-81)+-0.398(0)+0.515(0)+-0.042(132-137)+0.591(1)+0.477(1)+$ $0.056(12)+0.397(1)$

Individual estimated risk of $\operatorname{MRH}(\%)=\left(1 / 1+\mathrm{e}^{-0.435}\right) * 100$

Mrs V's absolute risk of experiencing MRH requiring healthcare use over the next 8 weeks is estimated at $\mathbf{3 9 \%}$

Figure 1 Prime tool to calculate patient risk of experiencing MRH requiring healthcare use within 8 weeks following hospital discharge. ADR, adverse drug reaction; GP, general practitioner; MRH, medication-related harm.

three times greater than the inpatient or ambulatory setting. ${ }^{2} 153357$ Major strengths of the PRIME tool are that: (1) it was developed using multicentre prospective data identifying a robust outcome that has relevance to patients, practitioners and policy makers (ie, definite MRH requiring healthcare use), (2) candidate predictors were clinically derived with patient and carer views considered, (3) the variables in the tool are routinely collected inpatient data, (4) the tool shows potentially superior clinical utility compared with some current risk stratification approaches based on a decision curve analysis.

This tool could support the WHO's campaign to reduce $\mathrm{MRH}$ during transitions of care $^{1}$ by flagging high-risk patients to target medicines optimisation interventions, and enabling clinicians to more accurately evaluate the risks of polypharmacy for individual patients. Furthermore, the personalised information from the tool could inform more individualised discussions around medication safety and enhance patient autonomy. ${ }^{58}$

\section{Comparison with other studies}

There are no directly comparable MRH tools as this is the first developed for application to the transition of care following hospital discharge. Moreover, the PRIME tool calculates risk of MRH rather than solely $\mathrm{ADR}$, as is the case with many existing tools (see online supplementary file for summary of existing tools). The PRIME tool's discrimination and calibration ${ }^{49} 59$ are comparable with these existing tools (PRIME C-statistic 0.66 vs C-statistics of other tools, eg, $\mathrm{ADRROP}^{60}$ (0.59), GerontoNet ${ }^{61}(0.70)$, PADR-EC ${ }^{62} \quad(0.67)$, BADRI $^{63}(0.73)$, Trivalle $\left.{ }^{64}(0.70)\right)$. The PRIME tool displayed good calibration, while other tools to predict MRH have not reported this important measure, with exception of BADRI (Hosmer-Lemeshow $\mathrm{p}=0.757$, calibration plot not reported). ${ }^{2363}$

The PRIME tool provides an absolute patient risk of $\mathrm{MRH}$, whereas other tools indicate a relative patient risk based on scoring systems that round predictor ORs to the nearest integer. While there is merit in making a tool as simple as possible for routine clinical 


\section{Original research}

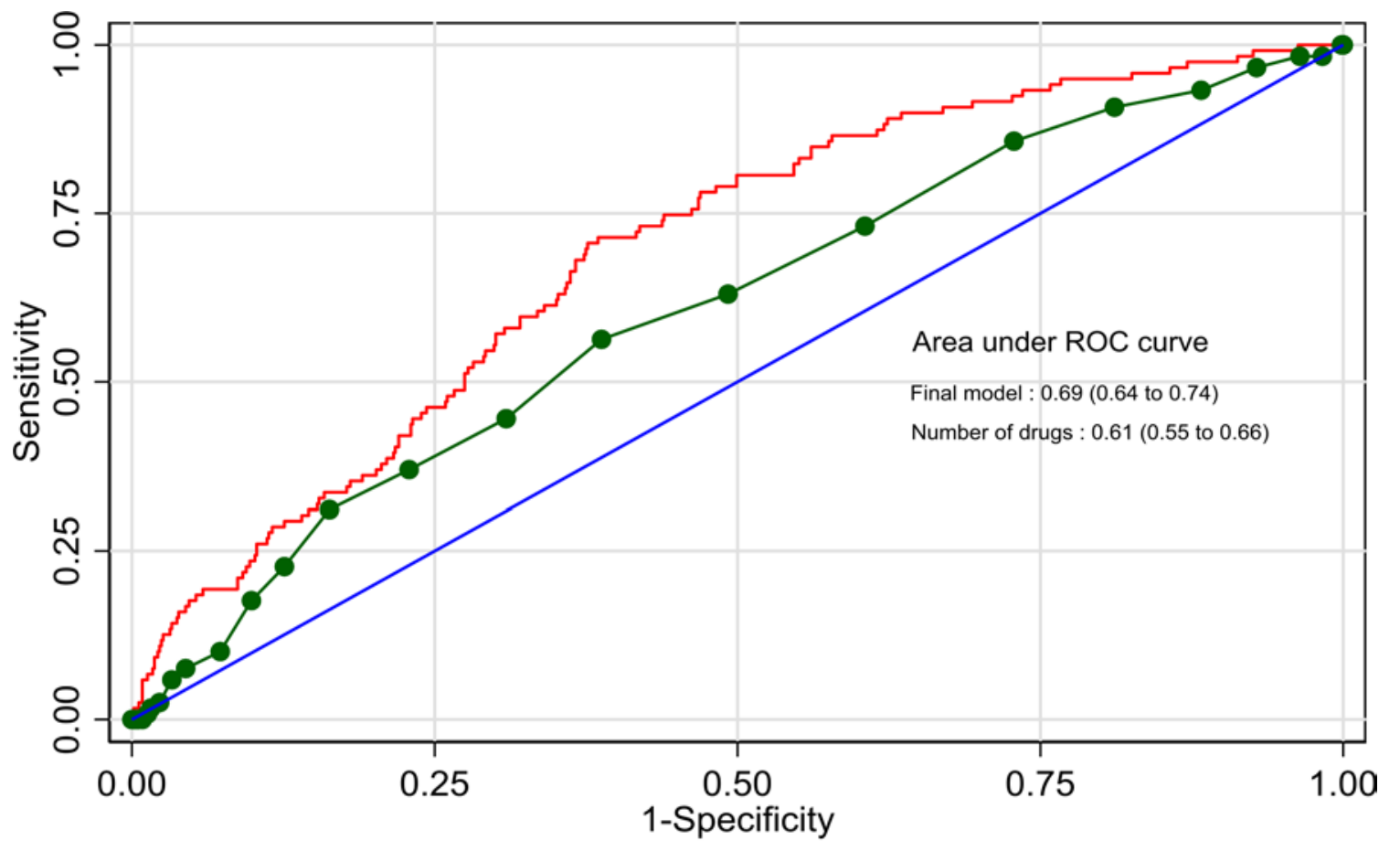

\section{Final Model \\ Reference}

Figure 2 Prime prediction tool compared with number of medicines alone to discriminate patient risk of medication harm. ROC, receiver operating characteristic.

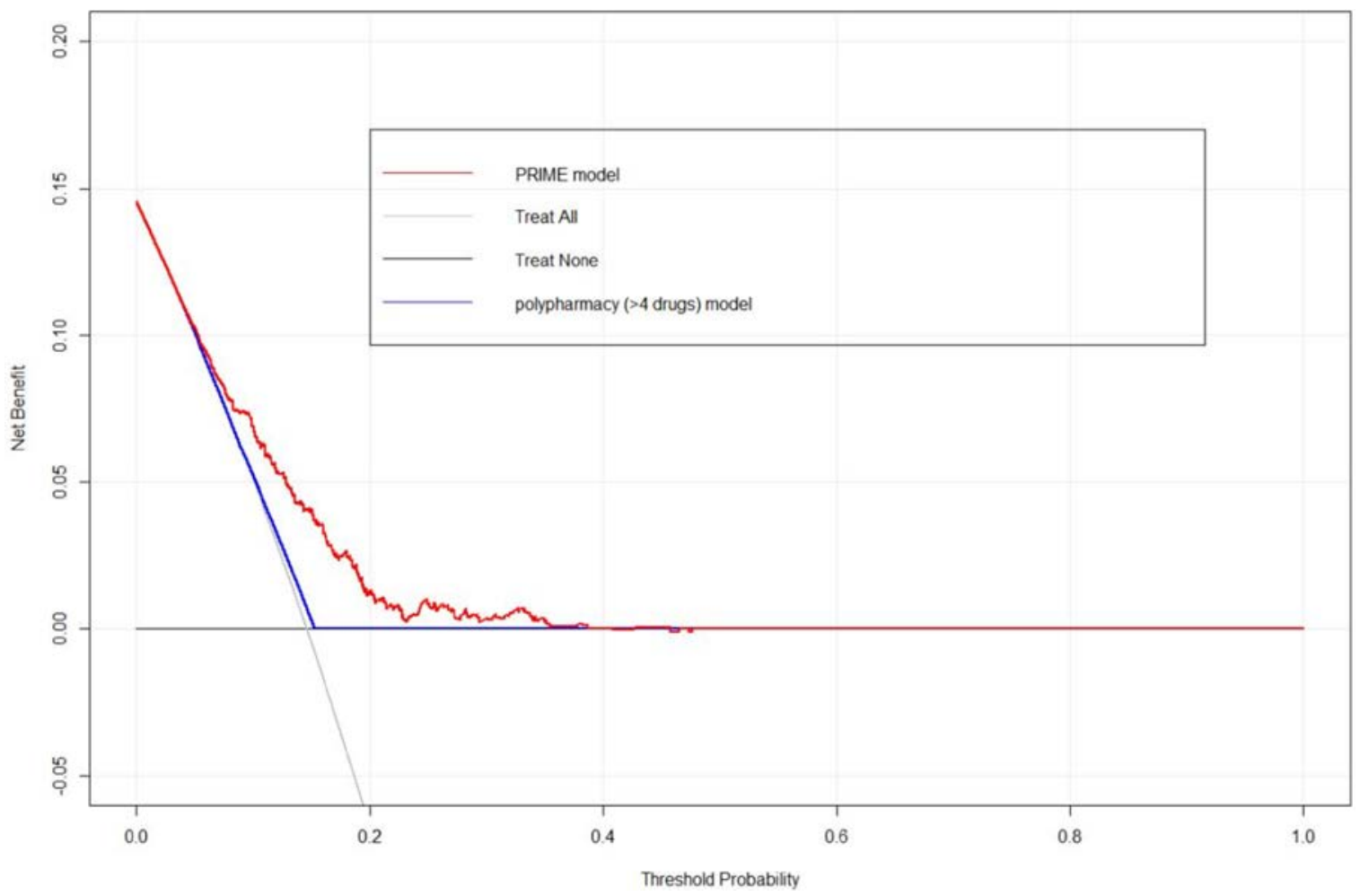

Figure 3 Decision curve comparing the net benefit of alternative models for clinical decision making. 
use, it can lead to imprecision of calculating patient risk. Given the widespread use of electronic patient records in the hospital setting, there is no longer a need to compromise the precision of risk calculation to facilitate manual calculations.

The decision curve analysis shows that the PRIME tool offers a higher net benefit compared with existing strategies to target individuals at increased risk of MRH. For instance, the MedSafer ${ }^{17}$ randomised controlled deprescribing trial includes older patients using more than four regular medicines to determine if a deprescribing intervention reduces adverse drug events 30 days postdischarge. The PRIME tool could offer an improved approach to targeting higher risk individuals for such interventions.

\section{Predictors in the model}

The PRIME tool includes a combination of demographic (age, female gender), medication-specific (number of medicines, antiplatelet drug, antidiabetic drug, past ADR), biochemical (sodium level) and social predictors (living alone). This comprehensive set of patient information reflects the complexity of predicting MRH and healthcare use. ${ }^{65}$ We anticipated the tool to hold a high degree of face validity among both clinicians and patients given that the selection of candidate variables was informed by both. Most of the variables in the tool have been previously demonstrated to be associated with MRH and/or unplanned healthcare utilisation in older adults. ${ }^{2}{ }^{616366-70} \mathrm{Few}$ studies have investigated living alone as a risk factor for $\mathrm{MRH}^{69}$ Our study substantiates self-reported evidence of adverse outcomes in older adults living alone following hospital discharge. ${ }^{71}$ The informal support from co-habiting family could facilitate medicine adherence and promote early recognition and management of MRH.

Our study extends the pool of evidence for poor outcomes associated with low sodium levels, including the postdischarge setting. ${ }^{6672-74}$ Even mild hyponatraemia is associated with attention deficits, postural instability and falls. ${ }^{73}$ This might contribute to patients being less receptive to medicines information around discharge, and less able to tolerate common ADRs such as dizziness without serious consequences.

Over time, patterns of prescribing will change and ongoing updates and validation of the tool is recommended, ${ }^{75}$ as has been the case for other tools. ${ }^{22}$ This might include investigating the impact of adding new prognostic factors, such as frailty $^{76}$ and genetic variants. $^{77}$

\section{Implications for practitioners and policy makers}

The PRIME tool is unique in providing practitioners with a patient's absolute risk of MRH following hospital discharge, and policy makers with a tool that identifies those that will consume healthcare resources as a consequence. While the tool has been developed to support a safer hospital discharge by better safeguarding patients at high risk of $\mathrm{MRH}$, the variables in the PRIME tool are routinely available and its usefulness could be evaluated in primary care settings.

The absence thus far of an evidence-based mechanism to risk stratify patients could have contributed to the limited effectiveness of previously tested interventions to reduce $\mathrm{MRH}$, in spite of strong face validity. ${ }^{1778-81}$ The PRIME tool could improve the efficiency and effectiveness of interventions to reduce MRH postdischarge. ${ }^{82}$ The integration of the tool into electronic discharge systems could automate the risk calculation and be made available to GPs on discharge summaries. Furthermore, the visibility of patient MRH risk to doctors compiling discharge summaries may stimulate wider positive changes in prescribing practices. For instance, it might prompt an additional check of the accuracy of the prescribed discharge medications given that a high proportion of discharge summaries convey inaccurate information of prescribed medicines. The availability of MRH risk information to GPs could stimulate stronger consideration of whether new patient symptoms are due to disease or MRH. With the advent of Primary Care Networks in the NHS, through which a major focus is prescribing and medicines safety, there is an opportunity to use the PRIME tool to prioritise the action of 7500 new clinical pharmacists being introduced into general practice under NHS England's $£ 100$ million medicines optimisation programme. $^{83}$

The use of probabilities is an important part of conveying health risk to patients in a comprehensible manner. ${ }^{84} 85$ Patients would co-own data on their risk of $\mathrm{MRH}$ at discharge, which might enhance their awareness that symptoms occurring in the initial weeks postdischarge might be due to MRH rather than the symptom of a new disease. Furthermore, the PRIME tool should aid better informed discussions between patients and doctors with regard to reducing polypharmacy, thus breaking down some barriers to deprescribing. ${ }^{86}$ Clinicians and patients tend to overestimate the benefits of treatments and underestimate the harms. ${ }^{87} 88$ Patients having individualised risk information could help to rebalance this skewed risk perception.

\section{Limitations}

There are several limitations of this work to be considered. Research pharmacists identifying MRH were not blinded to the study aim, potentially introducing bias. In cases of MRH leading to hospital re-admission, consultants independent of the study assigned causality to the event alongside the research pharmacist, thereby reducing the impact of potential bias. 
The PRIME tool was developed excluding patients with 'possible' and 'probable' MRH in order to strengthen the reliability of the model to detect true MRH cases in real-world clinical practice. This hypothesis needs to be tested in an external validation phase. Although bootstrap is an optimal method for internally validating a prognostic tool, it is not a replacement for the necessary external validation phase prior to implementation. The usability of the tool, once implemented into an electronic system, will also require investigation. Nonetheless, the large cohort investigated in this study using a multicentre, prospective design, in conjunction with a robust approach to $\mathrm{MRH}$ verification, and adjustment for model optimism, lends the PRIME tool to favourable re-testing.

Although the PRIME tool's C-statistic is not dissimilar to other tools for predicting complex outcomes, for example, stroke, ${ }^{89}$ certain cancers, ${ }^{90}$ and hospital readmission, ${ }^{91} 92$ the implications of false positives and false negatives should be considered. While a false positive may result in increased medication scrutiny, the information on risk has potential to cause unnecessary concern to patients and clinicians. Additionally, any intervention will use clinical resource that might have been better placed elsewhere in the healthcare system. False negatives meanwhile limit the tool's clinical utility to reduce $\mathrm{MRH}$ and avoidable healthcare use.

\section{CONCLUSIONS}

The PRIME tool identifies older patients at high risk of MRH requiring healthcare use following hospital discharge. This could support efforts to target medicines optimisation to reduce MRH and healthcare use. The tool should now be evaluated in new settings.

Contributors CR, RS, JH, GD, JMS, KA and TrdC conceived of the PRIME study. KA, JH, GD and CR were co-applicants for the study grant. NP, CR, GD, JMS and KA designed this study. Data analysis was performed by NP, KA, GD and CR and statistical expertise was provided by WB and SN. All authors were involved in preparing the manuscript. All authors reviewed and approved the final manuscript.

Funding This research was funded by the National Institute for Health Research (PB-PG-0711-25094).

Competing interests None declared.

Patient consent for publication Not required.

Ethics approval This research was approved by the National Research Ethics Service, East of England (REC Reference 13/ $\mathrm{EE} / 0075)$.

Provenance and peer review Not commissioned; externally peer reviewed.

Data availability statement All data relevant to the study are included in the article or uploaded as supplementary information.

Open access This is an open access article distributed in accordance with the Creative Commons Attribution Non Commercial (CC BY-NC 4.0) license, which permits others to distribute, remix, adapt, build upon this work non- commercially, and license their derivative works on different terms, provided the original work is properly cited, appropriate credit is given, any changes made indicated, and the use is noncommercial. See: http://creativecommons.org/licenses/by-nc/4. $0 /$.

ORCID iD

Nikesh Parekh http://orcid.org/0000-0002-9988-3717

\section{REFERENCES}

1 Donaldson LJ, Kelley ET, Dhingra-Kumar N, et al. Medication without harm: who's third global patient safety challenge.

Lancet 2017;389:1680-1.

2 Parekh N, Ali K, Page A, et al. Incidence of medication-related harm in older adults after hospital discharge: a systematic review. J Am Geriatr Soc 2018;66:1812-22.

3 World Health Organization. Medication safety in transitions of care. Geneva, 2019.

4 Davies EA, O'Mahony MS. Adverse drug reactions in special populations - the elderly. Br J Clin Pharmacol 2015;80:796-807.

5 Mangoni AA, Jackson SHD. Age-Related changes in pharmacokinetics and pharmacodynamics: basic principles and practical applications. Br J Clin Pharmacol 2003;57:6-14.

6 Gellad WF, Grenard JL, Marcum ZA. A systematic review of barriers to medication adherence in the elderly: looking beyond cost and regimen complexity. Am J Geriatr Pharmacother 2011.

7 Knight DA, Thompson D, Mathie E, et al. 'Seamless care? just a list would have helped!' older people and their carer's experiences of support with medication on discharge home from hospital. Health Expect 2013;16:277-91.

8 Mistiaen P, Duijnhouwer E, Wijkel D, et al. The problems of elderly people at home one week after discharge from an acute care setting. J Adv Nurs 1997;25:1233-40.

9 Mohammed MA, Moles RJ, Chen TF. Medication-related burden and patients' lived experience with medicine: a systematic review and metasynthesis of qualitative studies. BMJ Open 2016;6:e010035.

10 Krumholz HM. Post-Hospital syndrome - an acquired, transient condition of generalized risk. N Engl J Med 2013;368:100-2.

11 Cornu P, Steurbaut S, Leysen T, et al. Effect of medication reconciliation at hospital admission on medication discrepancies during hospitalization and at discharge. Int J Clin Pharm 2012;34:213-4.

12 Ensing HT, Koster ES, van Berkel PI, et al. Problems with continuity of care identified by community pharmacists postdischarge. J Clin Pharm Ther 2017;42:170-7.

13 Kattel S, Manning DM, Erwin PJ, et al. Information transfer at hospital discharge. J Patient Saf 2016;1.

14 Cornu P, Steurbaut S, Leysen T, et al. Discrepancies in medication information for the primary care physician and the geriatric patient at discharge. Ann Pharmacother 2012;46:983-91.

15 Parekh N, Ali K, Stevenson JM, et al. Incidence and cost of medication harm in older adults following hospital discharge: a multicentre prospective study in the UK. Br J Clin Pharmacol 2018;84:1789-97.

16 Vasilevskis EE, Shah AS, Hollingsworth EK, et al. A patientcentered deprescribing intervention for hospitalized older patients with polypharmacy: rationale and design of the 
Shed-MEDS randomized controlled trial. BMC Health Serv Res 2019;19:165.

17 McDonald EG, Wu PE, Rashidi B, et al. The MedSafer study: a controlled trial of an electronic decision support tool for deprescribing in acute care. J Am Geriatr Soc 2019;170.

18 Schnipper JL, Mixon A, Stein J, et al. Effects of a multifaceted medication reconciliation quality improvement intervention on patient safety: final results of the Marquis study. BMJ Qual Saf 2018;27:954-64.

19 England NHS. Next steps for risk stratification in the. London: NHS, 2015.

20 Kivlahan C, Webster AM, Health AV, et al. High-Risk-Patient identification: strategies for success, 2016.

21 Bitton A, Gaziano T. The Framingham heart study's impact on global risk assessment. Prog Cardiovasc Dis 2010;53:68-78.

22 Hippisley-Cox J, Coupland C, Brindle P. Development and validation of QRISK3 risk prediction algorithms to estimate future risk of cardiovascular disease: prospective cohort study. BMJ 2017;357.

23 Stevenson M, Williams L, Burnham G, et al. Predicting adverse drug reactions in older adults; a systematic review of the risk prediction models. Clin Interv Aging 2014;9.

24 Parekh N, Stevenson JM, Schiff R, et al. Can doctors identify older patients at risk of medication harm following hospital discharge? A multicentre prospective study in the UK. Br J Clin Pharmacol 2018;84:2344-51.

25 Mangin D, Bahat G, Golomb BA, et al. International Group for Reducing Inappropriate Medication Use \& Polypharmacy (IGRIMUP): Position Statement and 10 Recommendations for Action. Drugs Aging 2018:1-13.

26 Stevenson J, Parekh N, Ali K, et al. Protocol for a prospective (P) study to develop a model to stratify the risk (RI) of medication (M) related harm in hospitalized elderly (E) patients in the UK (the prime study). BMC Geriatr 2016;16.

27 Collins GS, Reitsma JB, Altman DG, et al. Transparent reporting of a multivariable prediction model for individual prognosis or diagnosis (TRIPOD): the TRIPOD statement. Ann Intern Med 2015;162.

28 Strand LM, Morley PC, Cipolle RJ, et al. DrugRelated problems: their structure and function. DICP 1990;24:1093-7.

29 Dormann H, Neubert A, Criegee-Rieck M, et al. Readmissions and adverse drug reactions in internal medicine: the economic impact. J Intern Med 2004;255:653-63.

30 Kellaway GS, McCrae E. Intensive monitoring for adverse drug effects in patients discharged from acute medical wards. $\mathrm{NZ}$ Med J 1973;78:525-8.

31 Kanaan AO, Donovan JL, Duchin NP, et al. Adverse drug events after hospital discharge in older adults: types, severity, and involvement of beers criteria medications. J Am Geriatr Soc 2013;61:1894-9.

32 Tangiisuran B, Davies JG, Wright JE, et al. Adverse drug reactions in a population of hospitalized very elderly patients. Drugs Aging 2012;29:669.

33 Hakkarainen KM, Gyllensten H, Jönsson AK, et al. Prevalence, nature and potential preventability of adverse drug events - a population-based medical record study of 4970 adults. Br J Clin Pharmacol 2014;78:170-83.

34 Hanlon JT, Pieper CF, Hajjar ER, et al. Incidence and predictors of all and preventable adverse drug reactions in frail elderly persons after hospital stay. J Gerontol A Biol Sci Med Sci 2006;61:511-5.
35 Naranjo CA, Busto U, Sellers EM, et al. A method for estimating the probability of adverse drug reactions. Clin Pharmacol Ther 1981;30:239-45.

36 Morisky DE, Green LW, Levine DM. Concurrent and predictive validity of a self-reported measure of medication adherence. Med Care 1986;24:67-74.

37 Parekh N, Gahagan B, Ward L, et al. 'They must help if the doctor gives them to you': a qualitative study of the older person's lived experience of medication-related problems. Age Ageing 2019;48:147-51.

38 Peduzzi P, Concato J, Kemper E, et al. A simulation study of the number of events per variable in logistic regression analysis. J Clin Epidemiol 1996;49:1373-9.

39 Steyerberg EW, Eijkemans MJC, Harrell FE, et al. Prognostic modeling with logistic regression analysis. Med Decis Mak 2001;21:45-56.

40 Falconer N, Barras M, Cottrell N. Systematic review of predictive risk models for adverse drug events in hospitalized patients. Br J Clin Pharmacol 2018;84:846-64.

41 van Smeden M, de Groot JAH, Moons KGM, et al. No rationale for 1 variable per 10 events criterion for binary logistic regression analysis. BMC Med Res Methodol 2016;16:163.

42 Vittinghoff E, McCulloch CE. Relaxing the rule of ten events per variable in logistic and COX regression. Am J Epidemiol 2007;165:710-8.

43 Carley S, Dosman S, Jones SR, et al. Simple nomograms to calculate sample size in diagnostic studies. Emerg Med J 2005;22:180-1.

44 Buderer NMF, methodology: S I. Statistical methodology: I. incorporating the prevalence of disease into the sample size calculation for sensitivity and specificity. Acad Emerg Med 1996;3:895-900.

45 White IR, Royston P, Wood AM. Multiple imputation using chained equations: issues and guidance for practice. Stat Med 2011;30:377-99.

46 Royston P, Moons KGM, Altman DG, et al. Prognosis and prognostic research: developing a prognostic model. BMJ 2009;338:b604.

47 Schmueli G. To explain or to predict? Stat Sci 2010;24:289-310.

48 Vatcheva KP, Lee M, Mccormick JB, et al. Multicollinearity in regression analyses conducted in epidemiologic studies HHS public access 2016.

49 Alba AC, Agoritsas T, Walsh M, et al. Discrimination and calibration of clinical prediction models. JAMA 2017;318.

50 Pavlou M, Ambler G, Seaman SR, et al. How to develop a more accurate risk prediction model when there are few events. BMJ 2015;351.

51 Austin PC, Tu JV. Bootstrap methods for developing predictive models. Am Stat 2004;58:7-137.

52 Smith GCS, Seaman SR, Wood AM, et al. Correcting for optimistic prediction in small data sets. Am J Epidemiol 2014;180:318-24.

53 Steyerberg EW, Harrell FE, Borsboom GJ, et al. Internal validation of predictive models: efficiency of some procedures for logistic regression analysis. J Clin Epidemiol 2001;54:774-81.

54 Vickers AJ, Van Calster B, Steyerberg EW. Net benefit approaches to the evaluation of prediction models, molecular markers, and diagnostic tests. BMJ 2016;352. 
55 Van Calster B, Wynants L, Verbeek JFM, et al. Reporting and interpreting decision curve analysis: a guide for Investigators. Eur Urol 2018;74:796-804.

56 Austin PC, Steyerberg EW, variable Eper. Events per variable (EPV) and the relative performance of different strategies for estimating the out-of-sample validity of logistic regression models. Stat Methods Med Res 2017;26:796-808.

57 Alhawassi M, Krass I, Bajorek V, et al. A systematic review of the prevalence and risk factors for adverse drug reactions in the elderly in the acute care setting. Clin Interv Aging $2079 ; 2014$.

58 Edwards A, Elwyn G. Understanding risk and lessons for clinical risk communication about treatment preferences. Qual Saf Heal Care 2001;10(Supplement 1):i9-13.

59 Steyerberg EW, Vickers AJ, Cook NR, et al. Assessing the performance of prediction models. Epidemiology 2010;21:128-38.

60 O'Mahony D, O'Connor MN, Eustace J, et al. The adverse drug reaction risk in older persons (ADRROP) prediction scale: derivation and prospective validation of an ADR risk assessment tool in older multi-morbid patients. Eur Geriatr Med 2018:1-9.

61 Onder G, Petrovic M, Tangiisuran B, et al. Development and validation of a score to assess risk of adverse drug reactions among in-hospital patients 65 years or older. Arch Intern Med $2010 ; 170$.

62 Parameswaran Nair N, Chalmers L, Connolly M, et al. Prediction of hospitalization due to adverse drug reactions in elderly community-dwelling patients (the PADR-EC score). PLoS One 2016;11:e0165757.

63 Tangiisuran B, Scutt G, Stevenson J, et al. Development and validation of a risk model for predicting adverse drug reactions in older people during hospital stay: Brighton adverse drug reactions risk (BADRI) model. PLoS One 2014;9:e111254.

64 Trivalle C, Burlaud A, Ducimetière P. Risk factors for adverse drug events in hospitalized elderly patients: a geriatric score. Eur Geriatr Med 2011;2:284-9.

65 Kaufmann CP, Stämpfli D, Hersberger KE, et al. Determination of risk factors for drug-related problems: a multidisciplinary triangulation process. BMJ Open 2015;5:e006376.

66 Donzé J, Aujesky D, Williams D, et al. Potentially avoidable 30-day Hospital readmissions in medical patients. JAMA Intern Med 2013;173.

67 Howard RL, Avery AJ, Slavenburg S, et al. Which drugs cause preventable admissions to hospital? A systematic review. $\mathrm{Br} J$ Clin Pharmacol 2007;63:136-47.

68 Budnitz D, Lovegrove MC, Shehab N, et al. Emergency hospitalizations for adverse drug events in older Americans. $N$ Engl J Med 2011;365:2002-12.

69 Malhotra S, Karan RS, Pandhi P, et al. Drug related medical emergencies in the elderly: role of adverse drug reactions and non-compliance. Postgrad Med J 2001;77.

70 Pimouguet C, Rizzuto D, Lagergren M, et al. Living alone and unplanned hospitalizations among older adults: a populationbased longitudinal study. Eur J Public Health 2016;27:ckw150.

71 Bragstad LK, Kirkevold M, Hofoss D, et al. Factors predicting a successful post-discharge outcome for individuals aged 80 years and over. Int J Integr Care 2012;12.

72 Wald Ret al. Impact of hospital-associated hyponatremia on selected outcomes. Arch Intern Med 2010;170:294.

73 Renneboog B, Musch W, Vandemergel X, et al. Mild chronic hyponatremia is associated with falls, unsteadiness, and attention deficits. Am J Med 2006;119:71.e1-71.e8.
74 Soiza R, Cumming K, Clarke J, et al. Hyponatremia: special considerations in older patients. JCM 2014;3:944-58.

75 Steyerberg EW, Moons KGM, van der Windt DA, et al. Prognosis research strategy (progress) 3: prognostic model research. PLoS Med 2013;10:e1001381.

76 Parekh N, Ali K, Stevenson JM, et al. Frailty predicts medication-related harm requiring healthcare: a UK multicentre prospective cohort study. Age Ageing 2019;48:i27-30.

77 Scutt G, Overall A, Bakrania P, et al. The Association of a Single-Nucleotide Polymorphism in the Nuclear Factor (Erythroid-Derived 2)-Like 2 Gene With Adverse Drug Reactions, Multimorbidity, and Frailty in Older People. $J$ Gerontol Ser A 2019;296.

78 Karapinar-Çarkıt F, van der Knaap R, Bouhannouch F, et al. Cost-Effectiveness of a transitional pharmaceutical care program for patients discharged from the hospital. PLoS One 2017;12:e0174513.

79 Thomas R, Huntley L, Mann M, et al. Pharmacist-Led interventions to reduce unplanned admissions for older people: a systematic review and meta-analysis of randomised controlled trials. Age Ageing 2014;43.

$80 \mathrm{McNab}$ D, Bowie P, Ross A, et al. Systematic review and meta-analysis of the effectiveness of pharmacist-led medication reconciliation in the community after hospital discharge. BMJ Qual Saf 2018;27:308-20.

81 Dhalla IA, O'Brien T, Morra D, et al. Effect of a Postdischarge virtual ward on readmission or death for high-risk patients. JAMA 2014;312.

82 Lai K, Howes K, Butterworth C, et al. Lewisham integrated medicines optimisation service: delivering a system-wide coordinated care model to support patients in the management of medicines to retain independence in their own home. Eur J Hosp Pharm 2015;22:98-101.

83 Bush J, Langley CA, Jenkins D, et al. Clinical pharmacists in general practice: an initial evaluation of activity in one English primary care organisation. Int J Pharm Pract 2017.

84 Calman KC. Communication of risk: choice, consent, and trust. The Lancet 2002;360:166-8.

85 Numeric LIM. Verbal, and visual formats of conveying health risks: suggested best practices and future recommendations. Med Decis Mak 2007;27:696-713.

86 Reeve E, To J, Hendrix I, et al. Patient barriers to and enablers of deprescribing: a systematic review. Drugs and Aging 2013;30:793-807.

87 Hoffmann TC DMC. Patients' Expectations of the Benefits and Harms of Treatments, Screening, and Tests. JAMA Intern Med $2015 ; 175$.

88 Hoffmann TC, Del Mar C. Clinicians' Expectations of the Benefits and Harms of Treatments, Screening, and Tests. JAMA Intern Med 2017;177:407.

89 GYH L. The CHA2DS2-VASc score for stroke risk stratification in patients with atrial fibrillation: a brief history. Eur Heart J 2015.

90 Pfeiffer RM, Park Y, Kreimer AR, et al. Risk prediction for breast, endometrial, and ovarian cancer in white women aged $50 \mathrm{Y}$ or older: derivation and validation from population-based cohort studies. PLoS Med 2013;10:e1001492.

91 van Walraven C, Dhalla IA, Bell C, et al. Derivation and validation of an index to predict early death or unplanned readmission after discharge from hospital to the community. CMAJ 2010;182:551-7. 
92 Billings J, Blunt I, Steventon A, et al. Development of a predictive model to identify inpatients at risk of re-admission within 30 days of discharge (PARR-30). BMJ Open 2012;2:e001667.

93 Roberts HC, Denison HJ, Martin HJ, et al. A review of the measurement of grip strength in clinical and epidemiological studies: towards a standardised approach. Age Ageing 2011;40:423-9.

94 Charlson ME, Pompei P, Ales KL, et al. A new method of classifying prognostic comorbidity in longitudinal studies: development and validation. J Chronic Dis 1987;40:373-83. 